2015 Global Fashion Management Conference at Florence Proceedings: 591-592 (June 2015) http://dx.doi.org/10.15444/GFMC2015.04.09.01

\title{
REPRODUCTION OF MULTI-COLOURED ARTWORK IN WOVEN FORM WITH REDEFINED SUBTRACTIVE COLOUR SYSTEMS
}

\author{
Ken Ri Kim, Kyung Hee University, Republic of Korea ${ }^{1)}$ \\ Chil Soon Kim, Kyung Hee University, Republic of Korea ${ }^{2}$
}

\begin{abstract}
This study enhances the capability to reproduce multi-coloured images in woven Jacquard forms where weave structure and pattern design were considerably involved in fabrication. In modern weaving, great convenience and efficiencies were established in both production and design process through digitalisation, while the colour adoption has been constrained as the applicable number of figuring yarns were limited. The enhancement toward colour realisation is traditionally related to weaving capability.

Surface colour display is dominated by additive, subtractive or optical mixing (Mathur, 2007). An additive colour system offers the largest gamut among output models yet, the light mixing principle is not suitable to apply to weave colour creation. Pre-dyed opaque yarns are used and juxtaposed; small particles of yarn colours reflect lights and they were observed as a certain form of colour. The common and crucial criterial pertinent to an optical mixing of weave colours were aligned more with the subtractive mixing principle.
\end{abstract}

A weave pattern was designed by subtractive primary colour classification and a multiweft figuring method. Secondary colours are theoretically produced when coupled CMY layers are mixed(i.e., cyan + magenta $=$ blue, cyan + yellow $=$ green, magenta + yellow $=$ red) and black is generated when all CMY primaries are mixed (Berns, 2000); however, non-bendable colours of threads are employed for colour reproduction in weaving and there is a limit to adopt a pigment mixing principle in the woven form. Therefore, each weave pattern required a modification to redefine primary colour regions and densities once an original artwork was separated and presented in greyscales levels. The weave patterns in an original subtractive scheme were altered by applying region-based segmentation to maximise the accessible colour gamut.

In this study, the weaving application developed for the multi-coloured image was introduced and the design process was explained based on a practical experiment proceeded with a newly developed weaving application.

Keywords: multi-coloured woven fabrication, subtractive colour system, weave pattern design, gradient colour deviation

\footnotetext{
${ }^{1)}$ kenrikim@hotmail.com

2) cskim@khu.ac.kr
} 


\section{References}

Berns, R. S., Billmeyer, F. W.,\& Saltzman, M.(3rd Eds.). (2000). Billmeyer and saltzman's principles of colour technology. New York, NY: Wiley.

Mathur, K., Donaldson, A., Hinks, D.,\&Seyam, A.M. (2005). Colour on demand for jacquard fabrics. Research Journal of Textile and Apparel, 9 (4), 26-37. 